\title{
BMJ Open Evaluation of actigraphy-measured sleep patterns among children with disabilities and associations with caregivers' educational attainment: results from a cross-sectional study
}

\author{
Xiaoli Chen, ${ }^{1}$ Juan Carlos Velez, ${ }^{2}$ Clarita Barbosa, ${ }^{2}$ Micah Pepper, ${ }^{2}$ Bizu Gelaye, ${ }^{1}$ \\ Susan Redline, ${ }^{1,3,4}$ Michelle A Williams ${ }^{1}$
}

To cite: Chen X, Velez JC, Barbosa C, et al. Evaluation of actigraphy-measured sleep patterns among children with disabilities and associations with caregivers' educational attainment: results from a cross-sectional study. BMJ Open 2015;5:e008589. doi:10.1136/bmjopen-2015008589

- Prepublication history and additional material is available. To view please visit the journal (http://dx.doi.org/ 10.1136/bmjopen-2015008589).

Received 24 April 2015 Revised 16 October 2015 Accepted 23 October 2015

CrossMark

For numbered affiliations see end of article.

Correspondence to Dr Xiaoli Chen; xchen@hsph.harvard.edu

\section{ABSTRACT}

Objectives: To use wrist-actrigrphy to collect objective measures of sleep and to characterise actigraphy-measured sleep patterns among children with disabilities. We also assessed the extent to which, if at all, caregivers' education is associated with children's sleep disturbances.

Design: Cross-sectional study.

Setting: A rehabilitation centre in the Patagonia region, Chile.

Methods: This study was conducted among 125 children aged 6-12 years with disabilities (boys: $55.2 \%$ ) and their primary caregivers in Chile. Children wore ActiSleep monitors for 7 days. A general linear model was fitted to generate least-square means and SEs of sleep efficiency (proportion of the sleep period spent asleep) across caregivers' education levels adjusting for children's age, sex, disability type, caregiver-child relationship and caregivers' age. Multivariable logistic regression analyses were conducted to estimate ORs and $95 \% \mathrm{Cls}$ of longer sleep latency $(\geq 30 \mathrm{~min})$ and longer wake after sleep onset (WASO) ( $\geq 90 \mathrm{~min}$ ) (a measure of sleep fragmentation) in relation to caregivers' educational attainment.

Results: Median sleep latency was $27.3 \mathrm{~min}$, WASO $88.1 \mathrm{~min}$ and sleep duration $8.0 \mathrm{~h}$. Mean sleep efficiency was $80.0 \%$. Caregivers' education was positively and significantly associated with children's sleep efficiency ( $p$ trend $<0.001$ ). Adjusted mean sleep efficiency was $75.7 \%$ ( $S E=1.4$ ) among children of caregivers <high school education, and $81.9 \%$ ( $\mathrm{SE}=1.0$ ) among children of caregivers >high school education. Compared to children whose caregivers had $>$ high school, children of caregivers with <high school had higher odds of longer sleep latency $(\mathrm{OR}=3.27$; $95 \% \mathrm{Cl} 1.12$ to 9.61 ) and longer WASO (OR=5.95; $95 \% \mathrm{Cl} 1.91$ to 18.53 ). Associations were consistent across disability types.

Conclusions: Children with disabilities experience difficulties initiating sleep (prolonged sleep latency) and maintaining sleep (long WASO, low sleep efficiency). Among children with disabilities, lower level

\section{Strengths and limitations of this study}

- This cross-sectional study is strengthened by its use of wrist-actigraphy which has been well validated for objective estimation of night-time sleep parameters in the natural sleep environment.

- Our study was unique in being the first study to examine the associations between caregivers' education and actigraphy-measured sleep among children with disabilities.

- Its strength also lies in the statistical analysis that was performed using univariate and multivariable linear and logistic regression models.

- This is a cross-sectional study with a small sample size and high heterogeneity of disabilities included.

of caregivers' education is associated with more sleep disturbances.

\section{INTRODUCTION}

Approximately 150 million children live with disabilities worldwide. ${ }^{1}$ Sleep disturbances, particularly difficulty initiating and maintaining sleep, are commonly reported among children with a wide range of disabilities including Down syndrome, attention-deficit/ hyperactivity disorder (ADHD), and autism. ${ }^{2-6}$ There are discrepancies between objective measures of sleep and those reported by parents of children with disabilities. ${ }^{5}$ Parents may be unaware of children's sleep disturbances, such as night awakenings, and tend to overestimate their children's sleep duration. ${ }^{7}$ Inferences from prior studies are limited because investigators have relied on parental report of child sleep. ${ }^{3}$ Although polysomnography is considered 
the gold standard for measuring sleep, it is burdensome and not easily amenable for use with children who have difficulties sleeping in unfamiliar surroundings. Actigraphy-based sleep offers opportunities to obtain objective measures of sleep in children's typical environment. Actigraphy has been validated for objective measures of sleep in clinical-based and population-based studies. $^{8}{ }^{9}$

Sleep disturbances have been related to sociodemographic, environmental and behavioural factors. ${ }^{10-13}$ Children's sleep disturbances have been connected with their home environment that may be influenced by caregivers' characteristics. ${ }^{13}{ }^{14}$ Our recent qualitative study showed that while Chilean caregivers and rehabilitation providers recognised the importance of sleep health, they differed in their understanding of how sleep practices influence children's sleep health. ${ }^{13}$ Parental ability to provide support for healthy behaviours including sleep may be influenced by parental education, a known factor associated with child health measures. ${ }^{12}$ 15-17 Magana et $a l^{15}$ reported that maternal education and knowledge about autism accounted for differences in the number of specialty services received by Latino children with autism spectrum disorder (ASD) as compared with Caucasian children in the USA. However, to our knowledge, little research has been conducted regarding the role of caregivers' education in objectively measured sleep among children with disabilities, particularly among Patagonian Chilean children, an understudied population with documented high burden of obesity and related chronic disorders. ${ }^{18}$

This cross-sectional study was designed to use wrist-actigraphy as a means for collecting objective measures of sleep patterns among children with disabilities. We also sought to characterise actigraphy-measured sleep patterns and to examine whether caregivers' education level is associated with sleep disturbances among children with disabilities. Specifically, we sought to estimate the prevalence of actigraphy-measured sleep disturbances including long sleep latency, long wake after sleep onset (WASO), short sleep duration, and poor sleep quality (low sleep efficiency) among Chilean children with disabilities. Further, following up on results from a previous qualitative study, ${ }^{13}$ we sought to determine the extent to which, if at all, caregivers' low levels of educational attainment are associated with children's sleep disturbances and overall poor sleep quality.

\section{METHODS}

\section{Participants}

The Chile Pediatric and Adult Sleep and Stress Study (CPASS) was a cross-sectional study that was established in September 2012 at the Centro de Rehabilitacion Club de Leones Cruz del Sur in Punta Arenas, Chile. ${ }^{13}$ The CPASS was designed to use wrist-actigraphy to characterise sleep patterns of children with disabilities. Particular attention was paid to establishing the protocols for data collection, assessing protocol adherence among children with a wide range of developmental disabilities, and using collected data to preliminary assess study hypotheses.

The first wave of the CPASS (CPASS I) was conducted between September and December in 2012 among children aged 6-12 years who were receiving routine clinical care for disabilities at the centre and their primary caregivers. Children with disabilities in this study were those who had impairments, activity limitations and participation restrictions due to health conditions such as autism, Down syndrome, and cerebral palsy, according to the WHO, the International Classification of Functioning, Disability and Health for Children and Youth (ICF-CY). ${ }^{19}$ All children were diagnosed by a clinical care team composed of a pediatric neurologist, a pediatrician with extensive experience in developmental disabilities, and a rehabilitation medicine specialist using diagnostic criteria and/or standardised tests with the input of families and teachers. Primary caregivers were parents, grandparents, relatives or other adults and were principally responsible for children's well-being and did not have developmental or intellectual disabilities. Research staff talked with caregivers to determine if they could complete the interviewer-administered questionnaire survey. In addition, a physician helped confirm that caregivers had no developmental or intellectual disabilities. Of 153 children whose families were contacted via telephone by research staff, 110 adult caregivers $(71.9 \%)$ consented to participate in this study. A total of 110 children and caregivers (one caregiver per child) were recruited in the CPASS I. Five children were excluded from this study due to fewer than 3 days of actigraphy data: four children removed monitors after day 1 or day 2 of the study; one child lost the monitor on day 2. These children had no sleep log data. A total of 105 child-caregiver dyads $(95.5 \%$ of enrolled families) completed the CPASS I study protocol.

The second wave of the CPASS (CPASS II) was conducted among children with disabilities aged 10 21 years and their primary caregivers between April and July in 2013 at the centre. ${ }^{20} 21$ Research personnel invited 129 caregivers of children with disabilities to participate. A total of 90 caregivers $(69.8 \%)$ agreed to participate in the study. Twenty children were aged 10-12 years from the CPASS II. As these two studies (CPASS I and CPASS II) were conducted between September 2012 and July 2013 at the same rehabilitation centre in Chile, with the same staff and with identical study protocols used for assessing sleep traits, we combined the results from the two studies in order to have a larger sample size with increased statistical power in the present analysis. In total, 125 children aged 6-12 years were included in the current study. The final sample size of this study was determined on the basis of available resources (eg, ActiSleep monitors) and not based on formal a priori sample size and statistical power determinations. 
Written informed consents were obtained from primary caregivers of children with disabilities. This study was approved by the institutional review boards of the Centro de Rehabilitacion Club de Leones Cruz del Sur and Harvard T.H. Chan School of Public Health Office of Human Research Administration.

\section{Study procedures}

A psychologist administered structured questionnaires to caregivers to collect information on sociodemographic and lifestyle factors of children and caregivers. Research staff instructed children to wear ActiSleep monitors (ActiLife, ActiGraph R\&D, Florida, USA) ${ }^{22}$ on their nondominant wrists for seven consecutive days. Although the monitors are waterproof, children were instructed to remove them from their wrists before taking showers and/or before swimming. Caregivers were also instructed to keep sleep logs of time in bed and time out of bed for their children. Electronic medical records were reviewed by a physician in order to extract children's diagnoses defined by the 10th revision of the International Statistical Classification of Diseases and Related Health Problems (ICD-10). ${ }^{23}$

\section{Sleep parameters}

Actigraphic sleep data were collected using the ActiSleep monitors and were analysed using the ActiLife 6 data analysis software. ${ }^{22}$ When worn during sleep episodes, the ActiSleep monitor can provide estimates of sleep onset, sleep latency, WASO, number and length of awakenings, sleep duration and sleep efficiency. Sleep latency is the length of time taken to fall asleep, calculated as the time between 'lights off' to the first period of 3 min of consecutive epochs scored as sleep. WASO is the number of minutes awake between sleep onset and time of final waking. Sleep efficiency is defined as the proportion of the estimated sleep periods spent asleep. Actigraphy data were collected in 1 min epochs using the zero-crossing modes, and the 'Sadeh' sleep algorithm was used for children. ${ }^{24}$ The majority of children $(\mathrm{n}=122,97.6 \%)$ had complete 7-day actigraphic sleep data; two children had 6-day actigraphic data and one child had 3-day data available for study. The averages of sleep parameters were calculated for children who had 3, 6 or 7 days of actigraphic data. There were no missing sleep log data for children included in this study. Caregivers were asked to record their children's sleep logs each morning up awakening and to make sure the sleep logs were complete. Research staff checked children's sleep logs when caregivers returned with ActiSleep monitors. Information from sleep logs were not used to substitute missing actigraphy data.

\section{Sociodemographic characteristics and children's disabilities}

Interviewer-administered questionnaires were used to collect information on children's age, sex, daytime napping, medication use, caregiver-child relationship (eg, mother), caregivers' age, employment status and education level. Caregivers were asked to report the highest degree of education they had completed: less than 12th grade (<high school education), high school graduate or equivalent (high school education), some college degree or college graduate or above (>high school education). According to the ICD-10, ${ }^{23}$ children's disabilities were grouped as: (1) mental and behavioural disorders (eg, ADHD, autism); (2) diseases of the musculoskeletal system and connective tissue, skin and subcutaneous tissue (eg, scoliosis); (3) diseases of the nervous system (eg, cerebral palsy); and (4) congenital malformations, deformations and chromosomal abnormalities (eg, Down syndrome).

\section{Statistical analysis}

Kolmogorov-Smirnov test was used to assess the normality of sleep parameters. Variables were described using means and SDs for parametric variables and medians and IQRs for non-parametric variables. Number of nocturnal awakenings and sleep efficiency were normally distributed, whereas sleep onset, sleep latency, WASO, awakening length and sleep duration were not normally distributed. Student's t test was used for age, number of awakenings, and sleep efficiency as parametric variables by child sex; Wilcoxon rank-sum test was used to test differences in non-parametric variables including sleep onset, sleep latency, WASO, awakening length and sleep duration by child sex. Fisher's or $\chi^{2}$ test was conducted to compare differences in sociodemographic factors, daytime napping, medication use and disability groups by child sex. Analysis of variance or Kruskal-Wallis test was conducted to evaluate the differences in sleep parameters across children's disability groups and caregivers' education levels.

A general linear model was fitted to generate least-square means and SEs of sleep efficiency across caregivers' education levels with adjustment for children's age, sex, disability, caregiver-child relationship, and caregivers' age. Univariate and multivariable linear regression analyses were performed to examine the associations of caregivers' education levels with sleep efficiency and the number of awakenings. Stratified analysis was conducted to evaluate whether the associations between caregivers' education levels and children's sleep disturbances differed according to children's disability groups. Given that we aimed to examine whether children whose caregivers had lower education level were more likely to have sleep disturbances (eg, long sleep latency, long WASO) and to enhance interpretability, we created categorical variables for these sleep parameters and used in logistic regression models. ORs and 95\% CIs were estimated using logistic regression models to evaluate the associations of caregivers' educational attainment with children's long sleep latency ( $\geq 30$ vs $<30 \mathrm{~min}$ ), long WASO ( $\geq 90$ vs $<90 \mathrm{~min}$ ) and short sleep duration ( $<8$ vs $\geq 8 \mathrm{~h}$ ). These cut points were chosen based on the literature of sleep latency ${ }^{25}$ and sleep duration, ${ }^{26}$ as well as 
sleep data distributions in the study (WASO median $=88$ min, sleep duration median $=8 \mathrm{~h}$; only $9.6 \%$ of children had sleep $\geq 9 \mathrm{~h}$ ). We also included a variable to represent study wave (I and II) in multivariable regression models and found similar results (data not shown). We conducted sensitivity analysis by including medication use and daytime napping in the models and did not find that they had an impact on our results in this study (data not shown). Statistical significance levels were set at $p$ $<0.05$ for two-sided analyses. $p$ Values were not corrected for multiple testing. All tests were performed using SAS V.9.3 (SAS Institute, Cary, North Carolina, USA).

\section{RESULTS}

The mean age of children with disabilities was 9.2 $(\mathrm{SD}=2.2$; range $=6-12)$ years. Most caregivers were mothers $(88.0 \%)$; $21.6 \%$ of caregivers reported having $<$ high school education level (table 1). There were no statistically significant differences in medication use, daytime napping, caregiver-child relationship, caregivers' age, employment status or education level between boys and girls.

Median time for sleep onset was 22:39, sleep latency $27.3 \mathrm{~min}$, WASO $88.1 \mathrm{~min}$ and sleep duration $8.0 \mathrm{~h}$ (table 1). The mean number of awakenings was 22.1 and sleep efficiency was $80.0 \%$. There were no statistically significant differences in sleep parameters between boys and girls (all $\mathrm{p}>0.05$ ). Overall, $43.2 \%$ of children had sleep latency $\geq 30 \mathrm{~min}, 51.2 \%$ had short sleep $<8 \mathrm{~h}$ and $77.6 \%$ had low sleep efficiency $<85 \%$.

There were no significant differences in sleep parameters across disability groups (see online supplementary figure S1), except for the number of nocturnal awakenings (see online supplementary table S1). Children with diseases of musculoskeletal system and connective tissue, skin and subcutaneous tissue appeared to have more awakenings, reflecting more fragmented sleep, than children with other disabilities.

Children whose caregivers had <high school had longer sleep latency, longer WASO, longer awakening length and lower sleep efficiency compared to children whose caregivers had $>$ high school (figure 1, table 2). Although children of caregivers with $<$ high school tended to have later sleep onset, higher number of awakenings, and shorter sleep duration when compared to children of caregivers with higher education, these differences did not reach statistical significance.

Our general linear model with adjustment for child age, sex, disability group, caregiver-child relationship and caregiver age showed that the mean sleep efficiency was the lowest among children of caregivers with $<$ high school $(75.7 \% \quad(\mathrm{SE}=1.4))$, followed by children of caregivers with high school $(80.4 \% \quad(\mathrm{SE}=1.0))$ and $>$ high school $(81.9 \%(\mathrm{SE}=1.0))$. Univariate and multivariable linear regression analyses showed no significant associations between caregivers' education levels and children's awakening number. However, compared to children whose caregivers had >high school, children of caregivers with $<$ high school had significantly lower sleep efficiency (adjusted $\beta=-6.3, \mathrm{SE}=1.7 ; \mathrm{p}=0.001$ ) (table 3). Caregivers' educational attainment was positively and significantly associated with children's sleep efficiency in the univariate model $(p$ trend $=0.001)$ and the multivariable model $(p$ trend $<0.001)$.

As shown in table 4 based on logistic regression models, compared to children whose caregivers had $>$ high school, children of caregivers with <high school had higher odds of longer sleep latency $\geq 30 \mathrm{~min}$ (adjusted $\mathrm{OR}=3.27$; 95\% CI 1.12 to 9.61 ) and longer WASO $\geq 90 \mathrm{~min} \quad(\mathrm{OR}=5.95 ; 95 \%$ CI 1.91 to 18.53$)$. Caregivers' education levels were inversely and significantly associated with children's sleep latency and WASO (both $\mathrm{p}$ trend $<0.05$ ). Children's short sleep duration $(<8 \mathrm{~h})$ was not associated with caregivers' low educational level (OR=0.78; 95\% CI 0.28 to 2.18 ).

The associations between caregivers' education and children's sleep efficiency varied little according to disability groups (data not shown in tables). For example, among children with mental and behavioural disorders, children of caregivers with $<$ high school had lower sleep efficiency $(\beta=-6.2, \mathrm{SE}=2.2 ; \mathrm{p}=0.006)$, after adjustment for children's age, sex, caregiver-child relationship and caregivers' age. Similar results were found for children with other disabilities.

\section{DISCUSSION}

In this cross-sectional study, we assessed sleep patterns using wrist actigraphy over seven consecutive days among Chilean children with disabilities. We found that actigraphy-measured sleep disturbances including long sleep latency (median=27 $\mathrm{min}$ ), long WASO (median $=88 \mathrm{~min}$ ), short sleep duration $($ median $=8 \mathrm{~h}$ ), high number of nocturnal awakenings $($ mean $=22)$ and low sleep efficiency $($ mean $=80 \%$ ) were common among children. These findings indicate that children with disabilities frequently experience difficulties initiating sleep (prolonged sleep latency) and maintaining sleep (long WASO, low sleep efficiency), and have increased sleep fragmentation (increased nocturnal awakenings). These indices are common in insomnia, which may occur secondary to chronic health conditions, and for many conditions, is associated with poor quality of life and increased disease-specific health burden including behavioural and cognitive problems. ${ }^{27}{ }^{28}$ We also found strong associations between caregivers' low educational attainment and children's sleep disturbances (eg, low sleep efficiency) independent of children's disability type and other covariates from children and caregivers. To our knowledge, this is the first study to specifically quantify actigraphy-measured sleep patterns among children with disabilities in relation to caregivers' education levels. Furthermore, we address these questions among Patagonian Chilean children with disabilities, an understudied population in South America. 
Table 1 Characteristics of children with disabilities and their primary caregivers

\begin{tabular}{|c|c|c|c|c|}
\hline & Total $(n=125)$ & Boys $(n=69)$ & Girls $(n=56)$ & p Value \\
\hline \multicolumn{5}{|l|}{ Child characteristics } \\
\hline Age, mean (SD), years & $9.2(2.2)$ & $8.8(2.1)$ & $9.8(2.3)$ & 0.015 \\
\hline \multicolumn{5}{|l|}{ Type of disability diagnosis, \% } \\
\hline Mental and behavioural disorders & 48.0 & 59.4 & 33.9 & \multirow[t]{4}{*}{0.004} \\
\hline Diseases of musculoskeletal system & 16.8 & 10.1 & 25.0 & \\
\hline Diseases of the nervous system & 27.2 & 27.5 & 26.8 & \\
\hline Congenital/chromosomal abnormalities & 8.0 & 2.9 & 14.3 & \\
\hline Daytime napping & 16.8 & 14.5 & 19.6 & 0.444 \\
\hline Medication use & 44.0 & 42.0 & 46.4 & 0.622 \\
\hline \multicolumn{5}{|l|}{ Sleep parameters } \\
\hline Sleep onset, median (IQR) & $22: 39(22: 06,23: 08)$ & $22: 44(22: 24,23: 08)$ & $22: 33(21: 54,23: 09)$ & 0.183 \\
\hline Sleep latency, median (IQR), minutes & $27.3(15.0,38.9)$ & $27.1(14.4,41.7)$ & $27.4(15.4,38.2)$ & 0.511 \\
\hline WASO, median (IQR), minutes & $88.1(65.6,111.1)$ & $92.4(64.4,112.4)$ & $81.9(66.2,107.0)$ & 0.411 \\
\hline Number of awakenings, mean (SD)* & $22.1(6.7)$ & $22.5(6.5)$ & $21.6(6.9)$ & 0.441 \\
\hline Awakening length, median (IQR), minutes & $5.1(4.2,7.1)$ & $5.1(4.2,7.0)$ & $5.1(4.2,7.1)$ & 0.923 \\
\hline Sleep duration, median (IQR), hours & $8.0(7.5,8.2)$ & $8.0(7.3,8.2)$ & $8.0(7.5,8.3)$ & 0.459 \\
\hline Sleep efficiency, mean (SD), \%* & $80.0(7.2)$ & $79.0(8.1)$ & $81.2(5.8)$ & 0.072 \\
\hline \multicolumn{5}{|l|}{ Caregiver characteristics } \\
\hline Age, mean (SD), years & $38.3(7.8)$ & $38.5(7.8)$ & $38.0(7.8)$ & 0.690 \\
\hline \multicolumn{5}{|l|}{ Caregiver-child relationship, \% } \\
\hline Mother & 88.0 & 88.4 & 87.5 & \multirow[t]{2}{*}{0.877} \\
\hline Other & 12.0 & 11.6 & 12.5 & \\
\hline Employed/self-employed & 60.0 & 62.3 & 57.1 & 0.557 \\
\hline \multicolumn{5}{|l|}{ Education level, \% } \\
\hline$<$ High school & 21.6 & 24.6 & 17.9 & \multirow[t]{3}{*}{0.088} \\
\hline High school & 37.6 & 29.0 & 48.2 & \\
\hline$>$ High school & 40.8 & 46.4 & 33.9 & \\
\hline
\end{tabular}


A

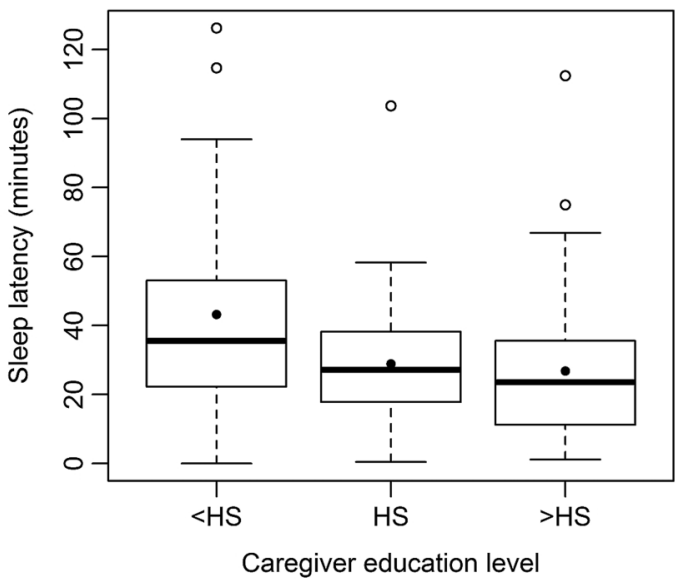

C

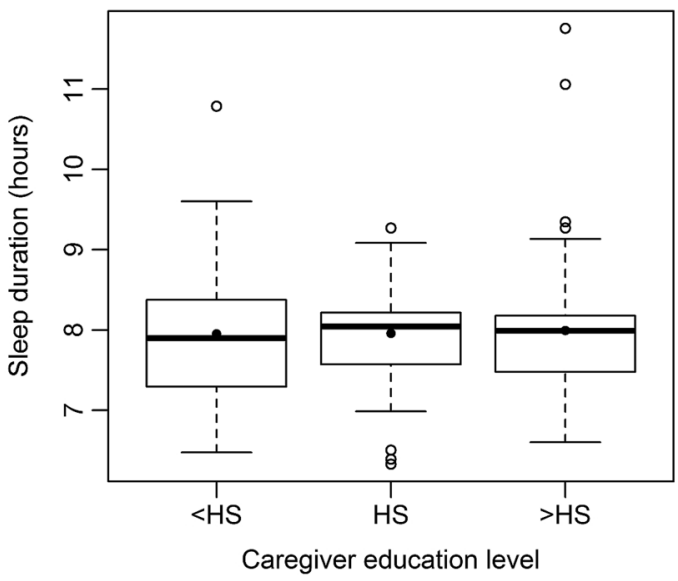

B

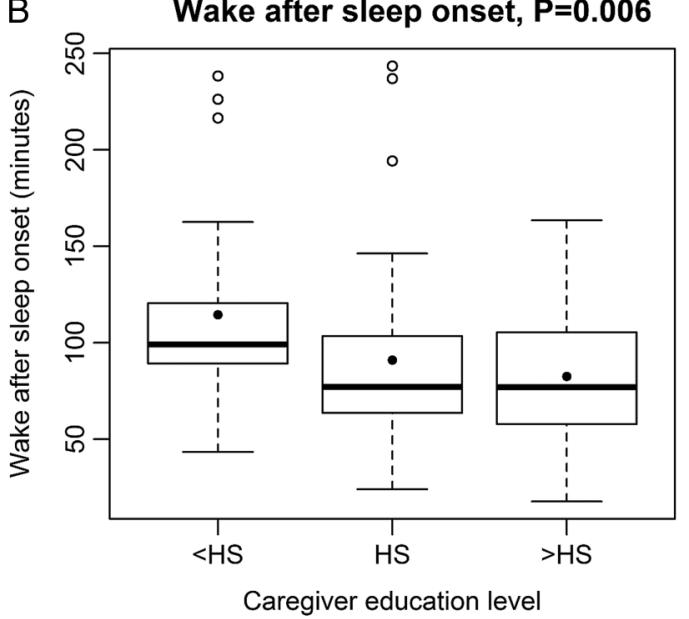

D Sleep efficiency, $P=0.002$

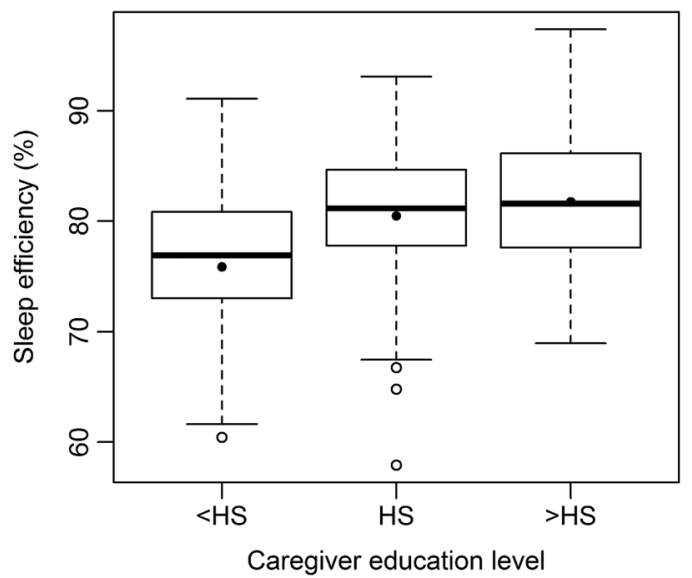

Figure 1 Children's sleep parameters, by caregiver education levels (HS: high school).

Children with a wide range of disabilities have been reported to have sleep disturbances, including prolonged sleep latency, increased WASO, short sleep duration and decreased sleep efficiency in Europe and other developed countries including Canada and New Zealand. ${ }^{4-6}{ }^{29}{ }^{30}$ For example, in a small study of 8 UK children with mucopolysaccharidosis aged 2-15 years, median actigraphy-measured sleep latency was $35.4 \mathrm{~min}$,
WASO $83.4 \mathrm{~min}$ and sleep efficiency was $75.6 \% .^{30}$ Allik et $a l^{6}$ reported that children aged 8-12 years with Asperger syndrome and high-functioning autism in Sweden had actigraphy-measured longer sleep latency (mean $=32$ minutes) and lower sleep efficiency (mean $=87 \%$ ) than controls. In a study of children aged 5-11 years with ADHD in Denmark, average sleep latency was $26 \mathrm{~min}$ and $31 \%$ of children had sleep

Table 2 Children's sleep parameters, by caregivers' education levels

\begin{tabular}{|c|c|c|c|c|}
\hline Sleep parameters & $\begin{array}{l}<\text { High school } \\
(n=27)\end{array}$ & $\begin{array}{l}\text { High school } \\
(n=47)\end{array}$ & $\begin{array}{l}>\text { High school } \\
(n=51)\end{array}$ & p Value \\
\hline Sleep onset, median (IQR) & 23:05 (22:17, 23:23) & $22: 31(21: 55,23: 04)$ & $22: 39(22: 24,23: 02)$ & 0.098 \\
\hline Sleep latency, median (IQR), minutes & $35.6(17.3,53.7)$ & $27.1(17.4,38.4)$ & $23.6(11.1,35.9)$ & 0.031 \\
\hline WASO, median (IQR), minutes & $99.1(88.1,121.0)$ & $77.1(63.4,103.4)$ & $77.0(57.6,106.0)$ & 0.006 \\
\hline Number of awakenings, mean (SD)* & $23.1(5.7)$ & $21.2(6.3)$ & $22.4(7.5)$ & 0.470 \\
\hline Awakening length, median (IQR), minutes & $6.9(5.0,9.5)$ & $5.2(4.2,7.2)$ & $4.7(3.9,5.7)$ & 0.001 \\
\hline Sleep duration, median (IQR), hours & $7.9(7.3,8.4)$ & $8.0(7.6,8.2)$ & $8.0(7.4,8.2)$ & 0.852 \\
\hline Sleep efficiency, mean (SD), \%* & $75.9(7.0)$ & $80.5(7.3)$ & $81.8(6.5)$ & 0.002 \\
\hline
\end{tabular}

*Sleep efficiency and number of awakenings were normally distributed in this study.

WASO, wake after sleep onset. 
Table 3 Linear regression models for associations between caregiver education and child sleep parameters

\begin{tabular}{|c|c|c|c|c|}
\hline & \multicolumn{2}{|c|}{ Number of awakenings } & \multicolumn{2}{|c|}{ Sleep efficiency (\%) } \\
\hline & $\beta$ (SE) & p Value & $\beta$ (SE) & p Value \\
\hline \multicolumn{5}{|l|}{ Univariate model } \\
\hline \multicolumn{5}{|c|}{ Caregiver education (ref: > high school) } \\
\hline High school & $-1.2(1.3)$ & 0.384 & $-1.3(1.4)$ & 0.357 \\
\hline$<$ High school & $0.7(1.6)$ & 0.662 & $-5.9(1.7)$ & $<0.001$ \\
\hline$p$ Value for trend test & & 0.838 & & 0.001 \\
\hline \multicolumn{5}{|l|}{ Multivariable model* ${ }^{*}$} \\
\hline \multicolumn{5}{|c|}{ Caregiver education (ref: >high school) } \\
\hline High school & $-0.6(1.4)$ & 0.674 & $-1.6(1.4)$ & 0.269 \\
\hline$<$ High school & $1.9(1.7)$ & 0.245 & $-6.3(1.7)$ & 0.001 \\
\hline$p$ Value for trend test & & 0.364 & & $<0.001$ \\
\hline
\end{tabular}

latency $>30$ min..$^{5}$ A case-control study using five nights of actigraphy for 15 school-aged children with traumatic brain injury and 15 school-aged siblings in the UK reported that brain injury was significantly associated with children's longer sleep latency (mean=50 $\mathrm{min}$ ), longer WASO (mean=65 $\mathrm{min}$ ) and lower sleep efficiency (mean=80\%). ${ }^{29}$ To our knowledge, no research has been conducted on objectively measured sleep parameters in South America (eg, Chile) among children with disabilities, an understudied sample. In our study of Chilean children with autism and other disabilities, the median of actigraphy-measured sleep latency was $27 \mathrm{~min}$, WASO was $88 \mathrm{~min}$, and the mean of sleep efficiency was $80 \%$, which were similar to previous research findings. ${ }^{52}{ }^{30}$ We also found that a high proportion of Chilean children with disabilities experienced actigraphy-measured sleep disturbances: $43 \%$ of children had sleep latency $\geq 30 \mathrm{~min}, 51 \%$ had short sleep $<8 \mathrm{~h}$ (90\% of children had sleep duration $<9 \mathrm{~h}$ ), and $78 \%$ had sleep efficiency $<85 \%$. Although school-aged children are recommended to have at least $9 \mathrm{~h}$ of sleep, ${ }^{26} 31$ in our study, only $10 \%$ of Chilean children with disabilities had sleep duration $\geq 9 \mathrm{~h}$. Gibbs $e t a l^{32}$ reported that among eight children aged 4-15 years with Prader-Willi syndrome in New Zealand, median 7-night actigraphymeasured WASO was $95 \mathrm{~min}$. Corkum et al $\mathrm{f}^{4}$ reported the average of awakenings was 15 among Canadian children with ADHD (aged 7-11 years). In our current study, the average number of nocturnal awakenings was 22 among Chilean children with ADHD and other disabilities. Our study along with previous research suggests that children with disabilities have difficulties initiating and maintaining good sleep.

Several researchers have reported that children with disabilities have late sleep onset. ${ }^{33}{ }^{34}$ A study of US children aged 2-5 years with autism and other developmental disabilities reported that the mean of 7-day actigraphy-measured sleep onset time was $21: 32 .{ }^{33}$ In this study, we found that median sleep onset time was 22:39 among Chilean children with autism and other disabilities. Unique climate (eg, late sunset) and lifestyle characteristics (eg, late mealtime) in the Patagonia region may have partly contributed to children's late sleep onset time. ${ }^{13} 18$ Our findings of late sleep onset are generally consistent with literature for children in this age range. For example, a cross-sectional study of 96 healthy Chilean children aged 10 years found that the mean of overnight polysomnography-measured sleep

Table 4 Logistic regression models for associations between caregiver education and child sleep disturbances

\begin{tabular}{|c|c|c|c|c|c|c|}
\hline & \multicolumn{2}{|c|}{ Sleep latency $\geq 30$ vs $<30 \mathrm{~min}$} & \multicolumn{2}{|c|}{ WASO $\geq 90$ vs $<90 \mathrm{~min}$} & \multicolumn{2}{|c|}{ Sleep duration $<8$ vs $\geq 8 \mathrm{~h}$} \\
\hline & OR (95\% Cl) & p Value & $\overline{\text { OR }(95 \% \mathrm{Cl})}$ & p Value & $\overline{\text { OR }(95 \% \mathrm{Cl})}$ & p Value \\
\hline \multicolumn{7}{|l|}{ Univariate model } \\
\hline \multicolumn{7}{|c|}{ Caregiver education (ref: > high school) } \\
\hline High school & $0.95(0.42$ to 2.17$)$ & 0.911 & $1.36(0.61$ to 3.04$)$ & 0.449 & $0.72(0.33$ to 1.60$)$ & 0.424 \\
\hline$<$ High school & 3.37 (1.26 to 8.98$)$ & 0.015 & $4.43(1.58$ to 12.38$)$ & 0.005 & 0.89 (0.35 to 2.25$)$ & 0.797 \\
\hline$p$ Value for trend test & & 0.029 & & 0.006 & & 0.689 \\
\hline \multicolumn{7}{|l|}{ Multivariable model } \\
\hline \multicolumn{7}{|c|}{ Caregiver education (ref: > high school)* } \\
\hline High school & $1.02(0.42$ to 2.45$)$ & 0.966 & $1.38(0.58$ to 3.30$)$ & 0.468 & $0.70(0.30$ to 1.64$)$ & 0.408 \\
\hline$<$ High school & 3.27 (1.12 to 9.61$)$ & 0.031 & 5.95 (1.91 to 18.53$)$ & 0.002 & 0.78 (0.28 to 2.18$)$ & 0.629 \\
\hline$p$ Value for trend test & & 0.056 & & 0.004 & & 0.526 \\
\hline
\end{tabular}


onset time was 23:10 for children with normal weight and 23:20 for children with overweight and obesity. ${ }^{35}$ Another study of 58 healthy children aged 11-13 years in the USA showed that the mean of 4-day actigraphymeasured sleep onset time was 23:27. ${ }^{36}$ In the Cleveland Children's Sleep and Health Study, $14.8 \%$ of US children aged 8-11 years had bedtime 23:00 or later. ${ }^{14}$ Future research is warranted to examine potential risk factors of late bedtime such as daytime napping and how to improve sleep onset time among children with disabilities.

It has been reported that children whose caregivers have low education levels are more likely to have adverse health outcomes, such as great body weight ${ }^{16}$ and poor quality of life. ${ }^{17}$ McDonald et $a l^{12}$ found that lower maternal education was independently associated with parent-reported shorter sleep $(<11 \mathrm{~h} /$ night $)$ among young children. Although several studies have reported that parental education is associated with child health, ${ }^{12}$ we are unaware of published reports that have investigated whether and how caregivers' education levels are related to objectively measured sleep problems among children with disabilities. In our study, caregivers' education level in relation to children's sleep disturbances was substantiated through statistically significant association as shown in univariate and multivariable linear and logistic regression models. Our study suggests the importance of tailoring sleep education for caregivers with lower educational attainment to improve children's sleep health. We considered low educational attainment a proxy for low socioeconomic status (SES). It has been reported that educational levels and SES are highly correlated. ${ }^{37}$ Low SES, as well as low education, has been related to high risk for cardiovascular disease, cancer and mortality. ${ }^{38}$ Parental SES has been inversely associated with negative child health outcomes including cardiovascular disease and mortality. ${ }^{39}{ }^{40}$ Some of the burden from low parental education level may be attributable to relatively lower health literacy, particularly sleep health literacy, which is pertinent to our observations in the focus group study ${ }^{13}$ and also in this epidemiological study.

Parents with low education levels may lack knowledge and resources about children's sleep practices and disabilities, resulting in adverse parenting practices known to affect sleep hygiene such as less limit setting, more variability in routines important to establish regular sleep patterns, suboptimal configuration of bedrooms to optimise sleep, inappropriate use of electronic devices, and meal timing in relationship to sleep. Better-educated parents may have more access to information about sleep and disabilities possibly through the internet or group membership with other parents of children diagnosed with disabilities. Future research should consider the impact of providing education and support such as sleep hygiene education, as well as initiatives to provide social support for parents and families of children with disabilities. Although our data suggest that these efforts may especially benefit less welleducated parents, all parents may have difficulties in navigating all of the controversies and different claims on the internet and may benefit from improved education and support. Our findings of the associations documented in our study population, if replicated, could help motivate the development and implementation of training programmes to increase the parental health literacy (especially sleep health literacy). Parents of children with disabilities could benefit from sleep education workshops. Social support from families of children with disabilities as well as healthcare providers including occupational and physiotherapists and nurses could also be important in helping parents handle with their children's sleep problems.

Lack of knowledge of healthy sleep behaviours among caregivers has been associated with an increased risk of having unhealthy sleep behaviours for their children. ${ }^{41}$ Although sleep disturbances have been noted to be common in children with neurological conditions (eg, cerebral palsy), there are no interventions specifically designed to improve sleep in these children. ${ }^{42}$ Several small studies of children with ASD have reported that parent-based sleep education appears effective in improving child sleep health. ${ }^{34} 43$ For example, a study of 20 children with ASD showed that parent-based sleep education workshops improved sleep latency from 62.2 min to 45.6 min. ${ }^{43}$ Malow $e t a l^{34}$ reported that sleep education to parents of 80 children with ASD (aged 2-10 years) significantly decreased children's sleep latency from 58.2 to $39.6 \mathrm{~min}$. Our prior qualitative study in Chile has indicated that parental knowledge gaps regarding healthy sleep behaviours in children support the need for increased sleep health education among targeted caregivers. ${ }^{13}$ Paediatricians and family physicians can be trained to provide adequate advice and educational messages (eg, sleep health) to paediatric patients and their families. ${ }^{44}$

Our study has several limitations. First, we did not have a comparison group of children without disabilities. Second, this study was limited by its modest sample size and the heterogeneity of disabilities represented among children studied. As such, the CIs were wider for some groups (eg, the less than high school education group) and we were not able to report associations specific to any single disability category (eg, autism). Third, we did not collect information pertaining to some aspects of sleep which may be associated with low education levels such as caregivers' shift work, sleep hygiene and other factors such as co-sleeping, lack of knowledge about sleep health, or inability to obtain medications to treat sleep or conditions in this study. Hence, we were not able to quantify the influence of these factors on children's sleep patterns. Our findings also focused on caregivers (majority were mothers) of children with disabilities in Chile, and may not be generalisable to other populations/groups. Although accepted to provide an objective measure of sleep-wake cycle, 
actigraphy has its limitations. For example, periods of quiet wakefulness may be interpreted by the device as sleep, and periods of restless sleep may be interpreted by the device as wakefulness. ${ }^{8}{ }^{45}$ Recent literature has indicated limitations of actigraphy for accurately capturing children's awakenings. ${ }^{46}$ Our study has indicated that children with diseases of the musculoskeletal system and connective, skin and subcutaneous tissue have more awakenings because their restless sleep may be represented by actigraphy as awakenings, and/or due to medication use or body pain. Despite this, actigraphy has been well validated for objective estimation of nocturnal sleep parameters across age groups in the natural sleep environment. ${ }^{9}$ As this was an exploratory study that aimed to examine whether parental education was associated with children's sleep disturbances, we did not adjust for multiple comparisons in this study. In addition, the simple adjustments for multiple comparisons might be overly conservative because many sleep problems (eg, long WASO, low sleep efficiency) were correlated.

\section{CONCLUSIONS}

We were successful in using wrist-actigraphy as a means of collecting objective measures of sleep patterns among Chilean Patagonian children with disabilities (eg, 97.6\% of children completed the 7-day data collection protocol). Using collected data, we noted that children with disabilities frequently experience difficulties initiating sleep (prolonged sleep latency), maintaining sleep (long WASO, low sleep efficiency) and sleep fragmentation (increased nocturnal awakenings). Among children with disabilities, lower levels of caregivers' educational attainment are strongly associated with children's sleep disturbances and these associations appear to be independent of children's age, sex, disability group, caregiver-child relationship and caregiver age. Parental support and education programmes directed to families with low education levels may be of particular importance for sleep behaviour intervention among children with disabilities. Larger studies, using wrist-actigraphy methods to objectively measure sleep in this relatively understudied population, are warranted to confirm our findings. Future research is also needed regarding the effects of sleep education intervention as well as social support to low educational families of children with disabilities on sleep health, with taking into account of different cultural backgrounds.

\section{Author affiliations \\ ${ }^{1}$ Department of Epidemiology, Harvard T.H. Chan School of Public Health, Boston, Massachusetts, USA \\ ${ }^{2}$ Centro de Rehabilitación Club de Leones Cruz del Sur, Punta Arenas, Chile ${ }^{3}$ Division of Sleep and Circadian Disorders, Brigham and Women's Hospital, Boston, Massachusetts, USA \\ ${ }^{4}$ Department of Medicine, Harvard Medical School, Boston, Massachusetts, USA}

Contributors All of the authors have agreed to the content of this manuscript. XC conceptualised and designed the study, supervised data collection, carried the initial analyses and drafted the initial manuscript. JCV and CB carried out the field survey, supervised data collection and critically reviewed and revised the manuscript. MP carried out the field survey, coordinated data collection at the study site and reviewed the manuscript. BG helped develop the study protocol, and critically reviewed and revised the manuscript. SR critically reviewed and revised the manuscript. MAW provided funding support to the study, supervised the study, conceptualised and designed the study and critically reviewed and revised the manuscript.

Funding This research was supported by awards from the National Institutes of Health (National Institute on Minority Health and Health Disparities: T37-MD001449), the National Center for Research Resources (NCRR)/the National Center for Advancing Translational Sciences (NCATS)

(8UL1TR000170-07), and the Rose Traveling Award.

\section{Competing interests None declared.}

\section{Patient consent Obtained.}

Ethics approval This study was approved by the institutional review boards of the Centro de Rehabilitacion Club de Leones Cruz del Sur and Harvard T.H. Chan School of Public Health Office of Human Research Administration.

Provenance and peer review Not commissioned; externally peer reviewed.

Data sharing statement No additional data are available.

Open Access This is an Open Access article distributed in accordance with the Creative Commons Attribution Non Commercial (CC BY-NC 4.0) license, which permits others to distribute, remix, adapt, build upon this work noncommercially, and license their derivative works on different terms, provided the original work is properly cited and the use is non-commercial. See: http:// creativecommons.org/licenses/by-nc/4.0/

\section{REFERENCES}

1. World report on disability. World Health Organization. 2011. http:// www.who.int/disabilities/ (accessed 20 Oct 2014).

2. Churchill SS, Kieckhefer GM, Landis CA, et al. Sleep measurement and monitoring in children with Down syndrome: a review of the literature, 1960-2010. Sleep Med Rev 2012;16:477-88.

3. Sung V, Hiscock $\mathrm{H}$, Sciberras E, et al. Sleep problems in children with attention-deficit/hyperactivity disorder: prevalence and the effect on the child and family. Arch Pediatr Adolesc Med 2008;162:336-42.

4. Corkum P, Tannock R, Moldofsky $\mathrm{H}$, et al. Actigraphy and parental ratings of sleep in children with attention-deficit/hyperactivity disorder (ADHD). Sleep 2001;24:303-12.

5. Hvolby A, Jorgensen J, Bilenberg N. Actigraphic and parental reports of sleep difficulties in children with attention-deficit/ hyperactivity disorder. Arch Pediatr Adolesc Med 2008;162:323-9.

6. Allik H, Larsson JO, Smedje H. Sleep patterns of school-age children with Asperger syndrome or high-functioning autism. J Autism Dev Disord 2006;36:585-95.

7. Sekine M, Chen X, Hamanishi S, et al. The validity of sleeping hours of healthy young children as reported by their parents. J Epidemiol 2002;12:237-42.

8. Sadeh A, Acebo C. The role of actigraphy in sleep medicine. Sleep Med Rev 2002;6:113-24.

9. Martin JL, Hakim AD. Wrist actigraphy. Chest 2011;139:1514-27.

10. Chen X, Wang R, Zee P, et al. Racial/Ethnic differences in sleep disturbances: the Multi-Ethnic Study of Atherosclerosis (MESA). Sleep 2015;38:877-88.

11. Mezick EJ, Matthews KA, Hall M, et al. Influence of race and socioeconomic status on sleep: Pittsburgh Sleep SCORE project. Psychosom Med 2008;70:410-16.

12. McDonald L, Wardle J, Llewellyn $\mathrm{CH}$, et al. Predictors of shorter sleep in early childhood. Sleep Med 2014;15:536-40.

13. Chen X, Gelaye B, Velez JC, et al. Attitudes, beliefs, and perceptions of caregivers and rehabilitation providers about disabled children's sleep health: a qualitative study. BMC Pediatr 2014; $14: 245$.

14. Spilsbury JC, Storfer-Isser A, Drotar D, et al. Effects of the home environment on school-aged children's sleep. Sleep 2005;28:1419-27.

15. Magana S, Lopez K, Aguinaga A, et al. Access to diagnosis and treatment services among latino children with autism spectrum disorders. Intellec Dev Disabil 2013;51:141-53.

16. Cameron AJ, van Stralen MM, Brug J, et al. Television in the bedroom and increased body weight: potential explanations for their 
relationship among European schoolchildren. Pediatr Obes 2013;8:130-41.

17. Kumar S, Kroon J, Lalloo R. A systematic review of the impact of parental socio-economic status and home environment characteristics on children's oral health related quality of life. Health Qual Life Outcomes 2014;12:41.

18. Velez JC, Fitzpatrick AL, Barbosa $\mathrm{Cl}$, et al. Nutritional status and obesity in children and young adults with disabilities in Punta Arenas, Patagonia, Chile. Int J Rehabil Res 2008;31:305-13.

19. International Classification of Functioning, Disability and Health (ICF). http://www.who.int/classifications/icf/en/ (accessed 16 Jul 2015)

20. Chen X, Gelaye B, Velez JC, et al. Caregivers' hair cortisol: a possible biomarker of chronic stress is associated with obesity measures among children with disabilities. BMC Pediatr 2015;15:9.

21. Chen X, Velez JC, Barbosa C, et al. Smoking and perceived stress in relation to short salivary telomere length among caregivers of children with disabilities. Stress 2015;18:20-8.

22. ActiLife 6. ActiLife, ActiGraph R\&D. http://www.theactigraph.com/ products/actisleep/ (accessed 12 Nov 2014).

23. International Classification of Diseases (ICD-10). ICD-10 International Statistical Classification of Diseases and Related Health Problems. 10th Revision. Volume 2. Instruction manual Published, 2010. http://www.icd10data.com/ICD10CM/Codes/ (accessed 16 Sep 2014).

24. Acebo C, Sadeh A, Seifer R, et al. Estimating sleep patterns with activity monitoring in children and adolescents: how many nights are necessary for reliable measures?. Sleep 1999;22:95-103.

25. Adkins KW, Molloy C, Weiss SK, et al. Effects of a standardized pamphlet on insomnia in children with autism spectrum disorders. Pediatrics 2012;130(Suppl 2):S139-144.

26. Chen X, Beydoun MA, Wang $Y$. Is sleep duration associated with childhood obesity? A systematic review and meta-analysis. Obesity (Silver Spring) 2008;16:265-74.

27. Lipton J, Becker RE, Kothare SV. Insomnia of childhood. Curr Opin Pediatr 2008;20:641-9.

28. Malow BA, Byars $\mathrm{K}$, Johnson $\mathrm{K}$, et al. A practice pathway for the identification, evaluation, and management of insomnia in children and adolescents with autism spectrum disorders. Pediatrics 2012;130(Suppl 2):S106-124.

29. Sumpter RE, Dorris L, Kelly T, et al. Pediatric sleep difficulties after moderate-severe traumatic brain injury. J Int Neuropsychol Soc 2013;19:829-34.

30. Mahon LV, Lomax M, Grant S, et al. Assessment of sleep in children with mucopolysaccharidosis type III. PLOS ONE 2014;9: e84128.

31. The National Sleep Foundation. http://sleepfoundation.org/ (accessed 12 Oct 2014).
32. Gibbs S, Wiltshire E, Elder D. Nocturnal sleep measured by actigraphy in children with Prader-Willi syndrome. J Pediatr 2013;162:765-9.

33. Goodlin-Jones BL, Tang K, Liu J, et al. Sleep patterns in preschool-age children with autism, developmental delay, and typical development. J Am Acad Child Adolesc Psychiatry 2008;47: 930-8.

34. Malow BA, Adkins KW, Reynolds A, et al. Parent-based sleep education for children with autism spectrum disorders. J Autism Dev Disord 2014;44:216-28.

35. Chamorro R, Algarin C, Garrido M, et al. Night time sleep macrostructure is altered in otherwise healthy 10-year-old overweight children. Int J Obes (Lond) 2014;38:1120-5.

36. Holm SM, Forbes EE, Ryan ND, et al. Reward-related brain function and sleep in pre/early pubertal and mid/late pubertal adolescents. $J$ Adolesc Health 2009;45:326-34.

37. Cassedy A, Drotar D, Ittenbach R, et al. The impact of socio-economic status on health related quality of life for children and adolescents with heart disease. Health Qual Life Outcomes 2013;11:99.

38. Vathesatogkit P, Batty GD, Woodward M. Socioeconomic disadvantage and disease-specific mortality in Asia: systematic review with meta-analysis of population-based cohort studies. $J$ Epidemiol Community Health 2014;68:375-83.

39. Ebrahim S, Montaner D, Lawlor DA. Clustering of risk factors and social class in childhood and adulthood in British women's heart and health study: cross sectional analysis. BMJ 2004;328:861.

40. Kim J, Son M, Kawachi I, et al. The extent and distribution of inequalities in childhood mortality by cause of death according to parental socioeconomic positions: a birth cohort study in South Korea. Soc Sci Med 2009;69:1116-26.

41. Owens JA, Jones C, Nash R. Caregivers' knowledge, behavior, and attitudes regarding healthy sleep in young children. J Clin Sleep Med 2011;7:345-50.

42. Galland BC, Elder DE, Taylor BJ. Interventions with a sleep outcome for children with cerebral palsy or a post-traumatic brain injury: a systematic review. Sleep Med Rev 2012;16:561-73.

43. Reed HE, McGrew SG, Artibee K, et al. Parent-based sleep education workshops in autism. J Child Neurol 2009;24:936-45.

44. Halal CS, Nunes ML. Education in children's sleep hygiene: which approaches are effective? A systematic review. J Pediatr 2014:90:449-56.

45. Johnson KP, Giannotti F, Cortesi F. Sleep patterns in autism spectrum disorders. Child Adolesc Psychiatr Clin N Am 2009;18:917-28.

46. Meltzer LJ, Walsh CM, Traylor J, et al. Direct comparison of two new actigraphs and polysomnography in children and adolescents. Sleep 2012;35:159-66. 